\title{
Asymmetric Electrophilic Difluoromethylthiolation of Indanone-Based $\beta$-Keto Esters Using Difluoromethanesulfonyl Hypervalent Iodonium Ylides
}

\author{
Satoshi Gondo ${ }^{1}$, Okiya Matsubara ${ }^{1}$, Hélène Chachignon ${ }^{2}$, Yuji Sumii ${ }^{1}$, Dominique Cahard ${ }^{2}$ (1) \\ and Norio Shibata $1,3, *$ (D) \\ 1 Department of Nanopharmaceutical Sciences, Department of Life Science and Applied Chemistry, Nagoya \\ Institute of Technology, Gokiso, Showa-ku, Nagoya 466-8555, Japan; 29411074@stn.nitech.ac.jp (S.G.); \\ cjh11141@nitech.jp (O.M.); sumii.yuji@nitech.ac.jp (Y.S.) \\ 2 CNRS, UMR 6014 COBRA, Normandie Université, 1 Rue Tesnière, \\ F-76821 Mont-Saint-Aignan Cedex, France; helene.chachignon@insa-rouen.fr (H.C.); \\ dominique.cahard@univ-rouen.fr (D.C.) \\ 3 Institute of Advanced Fluorine-Containing Materials, Zhejiang Normal University, 688 Yingbin Avenue, \\ Jinhua 321004, China \\ * Correspondence: nozshiba@nitech.ac.jp; Tel./Fax: +81-52-735-7543
}

Received: 8 December 2018; Accepted: 3 January 2019; Published: 9 January 2019

\begin{abstract}
The first electrophilic diastereoselective direct introduction of the difluoromethylthio group is described. We used a chiral auxiliary-based approach to illustrate the versatility of our recently developed difluoromethanesulfonyl hypervalent iodonium ylide reagents for the difluoromethylthiolation of indanone-based $\beta$-keto esters. Chiral $\mathrm{SCF}_{2} \mathrm{H}$-featuring compounds were obtained in up to $93 \%$ ee value.
\end{abstract}

Keywords: fluorine; sulfur; asymmetric synthesis; hypervalent iodine

\section{Introduction}

In the field of organofluorine chemistry one of the major present concerns is the development of new methods for the construction of novel chemical scaffolds. In this vein, the combination of sulfur, carbon and fluorine atoms has given birth to emergent motifs, which include $\mathrm{SCF}_{3}, \mathrm{SCF}_{2} \mathrm{H}, \mathrm{SCF}_{2} \mathrm{FG}$ $\left(\mathrm{FG}=\mathrm{SO}_{2} \mathrm{Ar}, \mathrm{SAr}, \mathrm{PO}(\mathrm{OR})_{2}, \mathrm{COAr}, \mathrm{Rf}\right), \mathrm{SCFH}_{2}$ [1-4]. The most recurring motif is undoubtedly the $\mathrm{SCF}_{3}$ one, which use grew at an unprecedented rate in the past recent years. The $\mathrm{SCF}_{3}$ chemotype is encountered in several biologically active molecules, albeit virtually absent in marketed drugs. The reason it elicits such enthusiasm is the exceptional high lipophilicity of $\mathrm{SCF}_{3}$ molecules that confers a high potential in medicinal chemistry. Equally interesting, though less often investigated, is the $\mathrm{SCF}_{2} \mathrm{H}$ group that also possess high lipophilicity while acting as hydrogen-bond donor owing to the acidity of the hydrogen atom [5]. The synthesis of enantioenriched molecules featuring a $\mathrm{SCF}_{2} \mathrm{R}$ motif directly linked to the chiral center is an issue worth consideration in the context of designing new chiral drugs. Asymmetric synthesis of trifluoromethylthiolated compounds have been investigated [6-22], and very recently Shibata and co-workers published the asymmetric synthesis of $\alpha$-tri- and difluoromethylthio allyl ketones via electrophilic difluoromethylthiolation of $\beta$-keto esters using difluoromethanesulfonyl hypervalent iodonium ylide 1a followed by a Pd-catalyzed Tsuji decarboxylative asymmetric allylic alkylation (DAAA, Scheme 1a) [23]. However, there is no report describing the asymmetric synthesis of difluoromethylthio compounds via a direct difluoro- methylthiolation reaction. Hence, we have been 
interested in direct asymmetric electrophilic difluoromethylthiolations. For this purpose, we targeted $\mathrm{SCF}_{2} \mathrm{H}$ analogues of $\alpha$-hydroxy $\beta$-keto esters, in particular those with an indanone scaffold [24-26] that are ubiquitous and important structural motifs, as such or in a masked form, in a wide range of biologically active natural products and synthetic pharmaceuticals and agrochemicals [27-34]. Moreover, we decided to valorize our difluoromethanesulfonyl hypervalent iodonium ylides $\mathbf{1 a , b}$ as electrophilic difluoromethyl- thiolation reagents for a wide range of nucleophiles [24]. Herein, we report the first asymmetric electrophilic introduction of the difluoromethylthio group onto chiral enamines derived from $\beta$-keto esters (Scheme $1 b$ ).

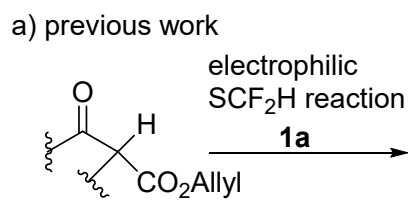

b) this work

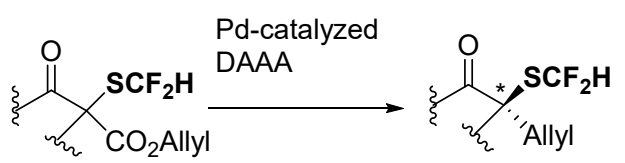

up to $94 \%$ ee<smiles>CCOC(=O)C(=CPc1ccccc1)C(=O)c1ccccc1</smiles>

$1 \mathbf{a}$

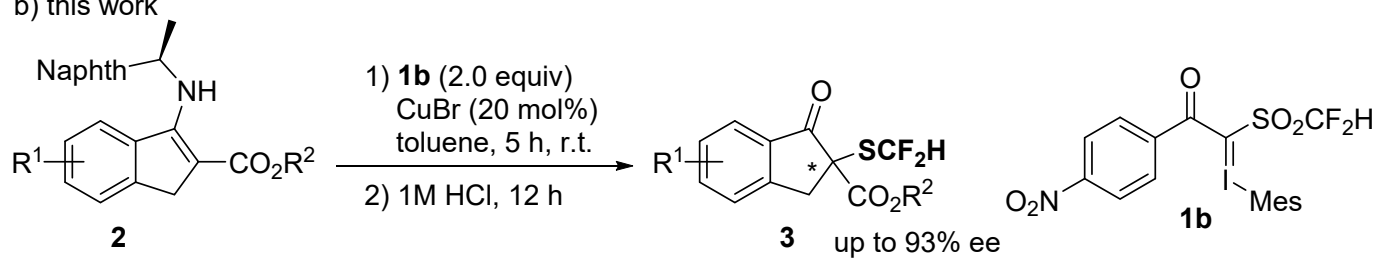

Scheme 1. Two methods for the asymmetric synthesis of difluoromethylthio-compounds with a tetrasubstituted carbon center; (a) previous work; (b) this work. The asterisk indicates the chiral center in the molecules.

\section{Results and Discussion}

We recently demonstrated that the difluoromethylthiolation of enamines obtained from $\beta$-keto esters was efficient and had wide generality [24], thus we surmised that the enamine approach would nicely extend to chiral enamines. In a first series of experiments, we studied the difluoromethylthiolation of $\beta$-enamino esters 2 a-e prepared from methyl 6-methyl-1-indanone-2- carboxylate and various chiral amines in order to determine the most appropriate chiral auxiliary (Table 1). The optimized reaction conditions found for the difluoromethylthiolation of achiral $\beta$-enamino esters were first applied to chiral $\beta$-enamino esters $2 \mathbf{a}-\mathbf{e}$. In the presence of $20 \mathrm{~mol} \%$ of copper bromide, the difluoromethanesulfonyl hypervalent iodonium ylide $\mathbf{1 b}$ reacted in 1,4 -dioxane at room temperature for $5 \mathrm{~h}$ followed by acidic cleavage of the resulting imine product to afford the 2-difluoromethylthio-1,3-dicarbonyl compound 3. The (S)-(-)- $\alpha$-methylbenzylamine auxiliary gave the desired product 3 in good yield and an encouraging 57\% ee value (Table 1, entry 1). Variations of the Ar and R groups of the chiral amine indicated that the bulkier (S)-(-)-1-(1-naphthyl)ethylamine led to enhanced enantioselectivity (Table 1, entry 3 ) albeit in a lower chemical yield. 
Table 1. Screening of chiral amines.

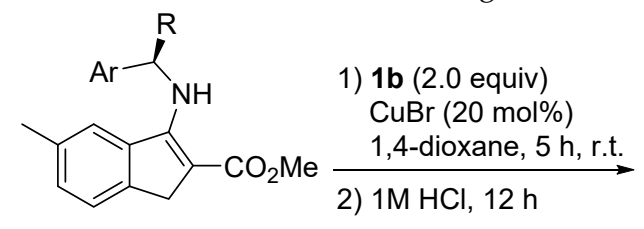

2<smiles>COC(=O)C1(SC(F)(F)F)Cc2ccc(I)cc2C1=O</smiles>

$3 a$

\begin{tabular}{cccccc}
\hline Entry & $\mathbf{A r}$ & $\mathbf{R}$ & $\mathbf{2}$ & Yield (\%) $^{\mathbf{1}}$ & Ee (\%) $^{\mathbf{2}}$ \\
\hline 1 & $\mathrm{Ph}$ & $\mathrm{Me}$ & $\mathbf{2 a}$ & 79 & 57 \\
2 & $\mathrm{Ph}$ & $\mathrm{Et}$ & $\mathbf{2 b}$ & 79 & 46 \\
3 & $1-\mathrm{Naphthyl}$ & $\mathrm{Me}$ & $\mathbf{2 c}$ & 57 & 62 \\
4 & $4-\mathrm{MeC}_{6} \mathrm{H}_{4}$ & $\mathrm{Me}$ & $\mathbf{2 d}$ & 80 & 57 \\
5 & $4-\mathrm{MeOC}_{6} \mathrm{H}_{4}$ & $\mathrm{Me}$ & $\mathbf{2 e}$ & 78 & 49 \\
\hline
\end{tabular}

${ }^{1}$ Yields for isolated pure products. ${ }^{2}$ Ee values were determined by HPLC analysis [35]. The asterisk indicates the chiral center in the molecule $3 a$.

Next, we conducted a second series of experiments in order to optimize the reaction solvent with substrates $\mathbf{2 a}$ or $\mathbf{2 c}$. Solvent screening revealed an increase in enantioselectivity in going from ether-containing solvents (1,4-dioxane, THF), chlorinated solvents $\left(\mathrm{CH}_{2} \mathrm{Cl}_{2}, \mathrm{CHCl}_{3}\right)$ to aromatic toluene, which provided the highest ee values of 69 and $88 \%$ ee, respectively, for both phenyl and naphthyl-based auxiliaries (Table 2). A survey of other parameters that are the amount and the nature $(\mathbf{a}$ versus $\mathbf{1 b})$ of the difluoromethanesulfenylating reagent and the amount and nature of the copper catalyst was also performed but deviation from standard conditions did not allow to improve the reactivity nor the enantioselectivity. We further attempted the reaction of $\beta$-keto esters with $\mathbf{1 b}$ in the presence of a catalytic amount of chiral amine, $(R)-1$-(naphthalen-1-yl)ethan-1-amine, but the reaction did not proceed well giving $3 \mathbf{a}$ in a low yield $(<10 \%)$.

Table 2. Optimization of the reaction conditions.

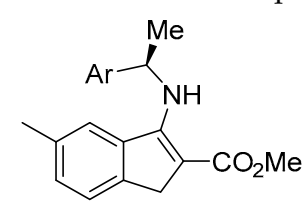

2

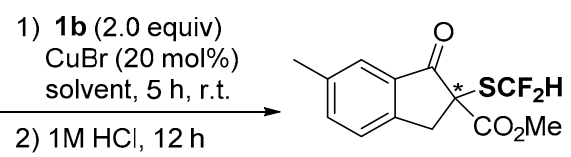

$3 \mathbf{a}$

\begin{tabular}{cccccc}
\hline Entry & $\mathbf{A r}$ & $\mathbf{2}$ & Solvent & Yield (\%) & Ee (\%) \\
\hline 1 & $\mathrm{Ph}$ & $\mathbf{2 a}$ & 1,4-dioxane & 79 & 57 \\
2 & $\mathrm{Ph}$ & $\mathbf{2 a}$ & $\mathrm{THF}$ & 75 & 56 \\
3 & $\mathrm{Ph}$ & $\mathbf{2 a}$ & $\mathrm{CH}_{2} \mathrm{Cl}_{2}$ & 74 & 67 \\
4 & $\mathrm{Ph}$ & $\mathbf{2 a}$ & $\mathrm{CHCl}_{3}$ & 75 & 68 \\
5 & $\mathrm{Ph}$ & $\mathbf{2 a}$ & $\mathrm{Tolluene}^{2}$ & 76 & 69 \\
6 & 1-Naphthyl & 2c & 1,4-dioxane & 57 & 62 \\
7 & 1-Naphthyl & 2c & $\mathrm{CH}_{2} \mathrm{Cl}_{2}$ & 52 & 88 \\
8 & 1-Naphthyl & 2c & $\mathrm{CHCl}_{3}$ & 65 & 87 \\
9 & 1-Naphthyl & 2c & Toluene & 59 & 88 \\
10 & 1-Naphthyl & 2c & $\mathrm{CH}_{3} \mathrm{CN}$ & 0 & - \\
\hline
\end{tabular}

${ }^{1}$ Yields for isolated pure products. ${ }^{2}$ Ee values were determined by HPLC analysis [35]. The asterisk indicates the chiral center in the molecule $3 \mathbf{a}$.

Having identified the suitable chiral auxiliary and the reaction conditions, we then turned our efforts to exploring other indanone-based enamine substrates featuring various substituents on the aromatic ring (Scheme 2). 


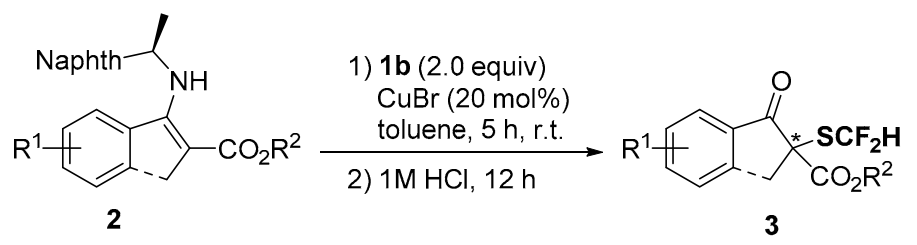

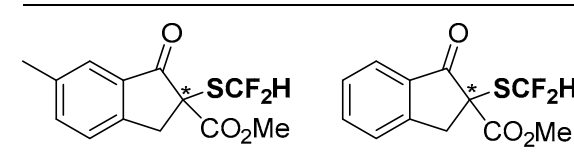

3a, $59 \%, 88 \%$ ee<smiles>CCCCC1(OC(C)=O)Cc2cc(Br)ccc2C1=O</smiles>

$3 \mathbf{e}, 32 \%, 85 \%$ ee 3b, $56 \%, 85 \%$ ee<smiles>CCSC1(OC)Cc2cc(F)ccc2C1=O</smiles>

3f, $63 \%, 86 \%$ ee<smiles>CCSC1(C(=O)OC)Cc2ccc(OC)cc2C1=O</smiles><smiles>CCSC1(C(OC)OC)Cc2cc(OC)c(OC)cc2C1=O</smiles>

$3 c, 51 \%, 90 \%$ ee 3d, $54 \%, 93 \%$ ee

Scheme 2. Difluoromethylthiolation of various indanone-based enamino esters and one acyclic enamino ester. The asterisk indicates the chiral center in the molecules 3.

We noticed that the enantiomeric excess increased for electron-donating substituents $(\mathrm{MeO}>\mathrm{Me}$ $>\mathrm{H}$ ) with a cumulative effect (two $\mathrm{MeO}>\mathrm{MeO}$, products $3 \mathbf{a}-\mathbf{d}$ ). Halogen substituted indanone-based enamines were compatible with the reaction conditions and gave similar ee values to the undecorated indanone (3e,f versus $\mathbf{3 b}$ ). The size of ester does not much affect the yield and enantioselectivity on the transformation ( $3 \mathbf{b}, \mathrm{R}^{2}=\mathrm{Me}$ and $\mathbf{3 g}, \mathrm{R}^{2}=\mathrm{Et}$, Scheme 2 ). In addition to these indanone carboxylates, we also attempted the substrates having six-membered tetralone-type structure and acyclic substrates. However, the tetralone-type substrate failed to deliver the corresponding $\beta$-enamino ester and an acyclic $\beta$-enamino ester produced a $\mathrm{SCF}_{2} \mathrm{H}$-product $3 \mathrm{~h}$ with a low ee $(12 \%$, see Scheme 2$)$. The chiral amine auxiliary was recovered in $25 \%$ yield after the reaction with $\mathbf{2} \mathbf{d}$ (not optimized) [36].

With regard to the reaction mechanism based on our previous reports [24,37], we proposed a copper-catalyzed generation of carbene $\mathbf{A}$ by reaction of the difluoromethanesulfonyl hypervalent iodonium ylide $\mathbf{1 b}$ followed by formation of the oxathiirene-2-oxide $\mathbf{B}$, which rearranged to the sulfoxide $\mathbf{C}$ and collapsed into the thioperoxoate $\mathbf{D}$. This $\mathrm{SCF}_{2} \mathrm{H}$ thioperoxoate was supposed to be the active electrophilic $\mathrm{HF}_{2} \mathrm{CS}^{+}$donor that reacted with the $\beta$-enamino esters 2 . The resulting iminium was then hydrolyzed under acidic conditions to release the desired $\alpha-\mathrm{SCF}_{2} \mathrm{H} \beta$-keto esters 3 (Scheme 3). 


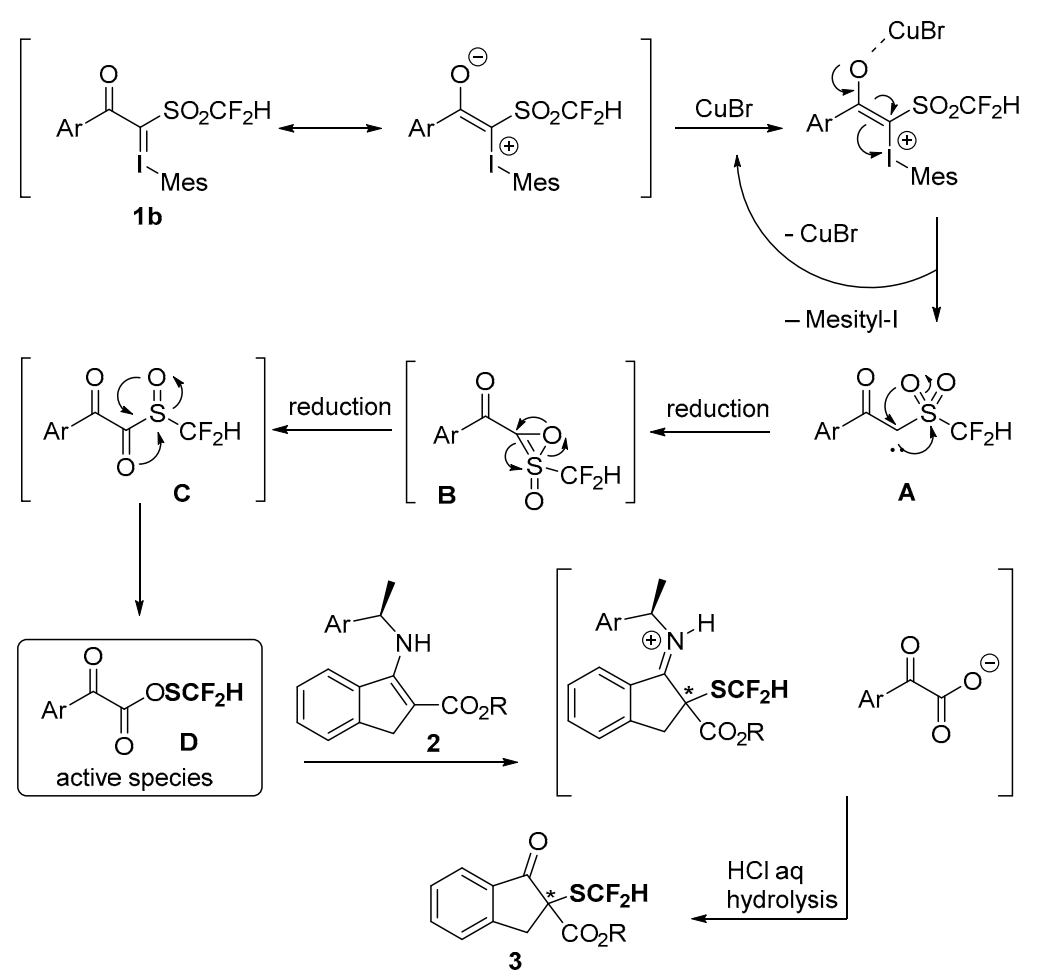

Scheme 3. Proposed reaction mechanism. The asterisk indicates the chiral center.

\section{Materials and Methods}

\subsection{General Information}

All reagents were used as received from commercial sources, unless specified otherwise. Enamino esters were prepared referring to previously reported procedures [37-39]. Reactions requiring anhydrous conditions were performed in flame-dried glassware under a positive pressure of nitrogen. Reaction mixtures were stirred magnetically. Solvents were transferred via syringe and were introduced into the reaction vessels though a rubber septum. All of the reactions were monitored by thin-layer chromatography (TLC) carried out on $0.25 \mathrm{~mm}$ silica-gel $\left(60-\mathrm{F}_{254}\right)$ (Merck, Kenilworth, NJ, USA). The TLC plates were visualized with UV light and 7\% phosphomolybdic acid or $\mathrm{KMnO}_{4}$ in water/heat. Preparative thin-layer plates carried out on $2.0 \mathrm{~mm}$ Merck silica gel $\left(60-\mathrm{F}_{254}\right)$. Column chromatography was carried out on a column packed with silica-gel $60 \mathrm{~N}$ spherical neutral size 50-63 $\mu \mathrm{m}$. The ${ }^{1} \mathrm{H}-\mathrm{NMR}$ $(300,700 \mathrm{MHz}$ ) was recorded on a Varian Mercury 300 (Agilent Technologies, Palo Alto, CA, USA) or an ECZ-700R (JEOL Ltd, Tokyo, Japan) instrument, with TMS ( $\delta=0.00 \mathrm{ppm})$ as internal standard, and ${ }^{19} \mathrm{~F}-\mathrm{NMR}(282 \mathrm{MHz})$ spectra was recorded on a Varian Mercury 300 with $\mathrm{C}_{6} \mathrm{~F}_{6}(\delta=-162.2 \mathrm{ppm})$ as internal standard. The ${ }^{13} \mathrm{C}-\mathrm{NMR}(125 \mathrm{MHz})$ spectra were recorded on an Avance 500 spectrometer (Bruker, Billerica, MA, USA). Chemical shifts $(\delta)$ are reported in parts per million and coupling constants $(J)$ are in hertz. The following abbreviations were used to show the multiplicities: s: singlet, $d$ : doublet, t: triplet, q: quadruplet, dd: doublet of doublets, td: triplet of doublets, dt: doublet of triplets, $\mathrm{m}$ : multiplet, br: broad. All the melting points are uncorrected. Mass spectra were recorded on an LCMS-2020EV (ESI-MS) system (Shimadzu Corporation, Kyoto, Japan). Infrared spectra were recorded on a FT/IR-4100 spectrometer (JASCO Corporation, Tokyo, Japan). HPLC analyses were performed on a JASCO PU-2080 Plus system using a $4.6 \times 250 \mathrm{~mm}$ CHIRALPAK IB-3 column and a CHIRALCEL OD-3 column. Optical rotations were measured on a SEPA-300 instrument (HORIBA Ltd, Kyoto, Japan). High resolution mass spectrometry were recorded on a Synapt G2 HDMS (ESI-MS) system (Waters Corporation, Milford, MA, USA). The chiral amines: $(S)-(-)$ - $\alpha$-methylbenzylamine $(\geq 99.5 \%$ ee), (S)-(-)- $\alpha$-ethylbenzylamine ( $\geq 99.0 \%$ ee), (S)-(-)-4-methoxy- $\alpha$-methylbenzylamine $(\geq 97.5 \%$ ee) were purchased from Sigma Aldrich (St. Louis, MI, USA). (S)-(-)-1-(1-Naphthyl)ethylamine (>98.0\% ee), 
and (S)-(-)-1-(p-tolyl)ethylamine were purchased from TCI (Tokyo, Japan). The ${ }^{1} \mathrm{H},{ }^{13} \mathrm{C}$ and ${ }^{19} \mathrm{~F}$ NMR spectra of compounds 3 and HPLC data of compounds 3 are available in the Supplementary Material.

\subsection{Synthesis of Chiral Enamine (General Procedure)}

Amine (2.2 mmol, 1.5 equiv) was added to a solution of $\beta$-ketoester $(1.47 \mathrm{mmol})$ and zinc acetate $(0.29 \mathrm{mmol}, 20 \mathrm{~mol} \%)$ in methanol under nitrogen atmosphere, the reaction mixture was refluxed for 16-64 h. After the reaction, the mixture was concentrated under reduced pressure, and the residue was purified by column chromatography (with ethyl acetate: hexane mixtures as eluent).

Methyl 5-methyl-3-((1-phenylethyl)amino)-1H-indene-2-carboxylate (2a). Following the general procedure the reaction mixture was stirred at $50{ }^{\circ} \mathrm{C}$ for $53 \mathrm{~h}$. After the reaction was complete, the mixture was worked up as described. Brown solid $(35 \%, 156.6 \mathrm{mg}) .{ }^{1} \mathrm{H}-\mathrm{NMR}\left(300 \mathrm{MHz}, \mathrm{CDCl}_{3}\right) \delta 8.29(\mathrm{~d}, J=6.3 \mathrm{~Hz}$, $1 \mathrm{H}), 7.21-7.43(\mathrm{~m}, 7 \mathrm{H}), 7.11(\mathrm{~d}, J=7.8 \mathrm{~Hz}, 1 \mathrm{H}), 5.36-5.41(\mathrm{~m}, 1 \mathrm{H}), 3.81(\mathrm{~s}, 3 \mathrm{H}), 3.50(\mathrm{~s}, 2 \mathrm{H}), 2.27(\mathrm{~s}, 3 \mathrm{H})$, $1.66(\mathrm{~d}, J=6.8 \mathrm{~Hz}, 3 \mathrm{H}) .{ }^{13} \mathrm{C}-\mathrm{NMR}\left(125 \mathrm{MHz}, \mathrm{CDCl}_{3}\right) \delta 21.5,26.0,33.9,50.4,53.8,97.2,124.3,124.5,125.4$ (2C), 127.0, 128.7 (2C), 129.2, 135.6, 137.6, 142.6, 145.3, 159.1, 168.8. IR (KBr): 3284, 2958, 2924, 1643, 1587, 1564, 1444, 1317, 1267, $1205 \mathrm{~cm}^{-1}$. MS (ESI): $\mathrm{m} / z 308(\mathrm{M}+\mathrm{H})^{+}$.

Methyl 5-methyl-3-((1-phenylpropyl)amino)-1H-indene-2-carboxylate (2b). Gray solid (30\%, $153.5 \mathrm{mg})$. ${ }^{1} \mathrm{H}-\mathrm{NMR}\left(300 \mathrm{MHz}, \mathrm{CDCl}_{3}\right) \delta 8.36(\mathrm{~d}, J=8.1 \mathrm{~Hz}, 1 \mathrm{H}), 7.19-7.40(\mathrm{~m}, 7 \mathrm{H}), 7.10(\mathrm{~d}, J=7.8 \mathrm{~Hz}, 1 \mathrm{H})$, 5.09-5.15 (m, 1H), $3.82(\mathrm{~s}, 3 \mathrm{H}), 3.49(\mathrm{~s}, 2 \mathrm{H}), 2.27(\mathrm{~s}, 3 \mathrm{H}), 1.90-1.99(\mathrm{~m}, 2 \mathrm{H}), 1.06(\mathrm{t}, J=7.1 \mathrm{~Hz}, 3 \mathrm{H})$. ${ }^{13} \mathrm{C}-\mathrm{NMR}\left(125 \mathrm{MHz}, \mathrm{CDCl}_{3}\right) \delta 10.7,21.5,32.8,33.9,50.3,59.8,97.1,124.3,124.5,126.0(2 \mathrm{C}), 126.9,128.6$ (2C), 129.2, 135.5, 137.6, 142.6, 144.0, 159.6, 168.9. IR (KBr): 3273, 2970, 2951, 1651, 1595, 1568, 1460 , $1309,1263,1194 \mathrm{~cm}^{-1}$. MS (ESI): $m / z 322(\mathrm{M}+\mathrm{H})^{+}$.

Methyl 5-methyl-3-((1-(naphthalen-1-yl)ethyl)amino)-1H-indene-2-carboxylate (2c). Pale yellow solid (40\%, $211.0 \mathrm{mg}) .{ }^{1} \mathrm{H}-\mathrm{NMR}\left(300 \mathrm{MHz}, \mathrm{CDCl}_{3}\right) \delta 8.48(\mathrm{~d}, J=6.3 \mathrm{~Hz}, 1 \mathrm{H}), 8.16(\mathrm{~d}, J=8.4 \mathrm{~Hz}, 1 \mathrm{H}), 7.89(\mathrm{~d}$, $J=8.1 \mathrm{~Hz}, 1 \mathrm{H}), 7.71(\mathrm{~d}, J=8.4 \mathrm{~Hz}, 1 \mathrm{H}), 7.60-7.66(\mathrm{~m}, 2 \mathrm{H}), 7.55-7.50(\mathrm{~m}, 1 \mathrm{H}), \delta 7.38(\mathrm{t}, J=7.7 \mathrm{~Hz}, 1 \mathrm{H})$, 7.20-7.24 (m, 1H), $7.10(\mathrm{~s}, 1 \mathrm{H}), 6.97(\mathrm{~d}, J=7.5 \mathrm{~Hz}, 1 \mathrm{H}), 6.09-6.14(\mathrm{~m}, 1 \mathrm{H}), 3.83(\mathrm{~s}, 3 \mathrm{H}), 3.49$ (d-like, 2H), $1.83(\mathrm{~s}, 3 \mathrm{H}), 1.78(\mathrm{~d}, J=6.6 \mathrm{~Hz}, 3 \mathrm{H}) .{ }^{13} \mathrm{C}-\mathrm{NMR}\left(125 \mathrm{MHz}, \mathrm{CDCl}_{3}\right) \delta 21.0,24.8,33.9,50.1,50.4,96.8,121.7$, 122.6, 124.1, 124.3, 125.5, 125.9, 126.4, 127.6, 129.1, 129.2, 129.7, 133.8, 135.3, 137.4, 140.9, 142.4, 159.0, 168.9. IR (KBr): 3296, 3059, 2885, 2862, 1655, 1591, 1564, 1448, 1267, $1186 \mathrm{~cm}^{-1}$. MS (ESI): $m / z 358$ $(\mathrm{M}+\mathrm{H})^{+}$.

Methyl 5-methyl-3-((1-(p-tolyl)ethyl)amino)-1H-indene-2-carboxylate (2d). Yellow solid (20\%, $96.6 \mathrm{mg})$. ${ }^{1} \mathrm{H}-\mathrm{NMR}\left(300 \mathrm{MHz}, \mathrm{CDCl}_{3}\right) \delta 8.26(\mathrm{~d}, J=6.9 \mathrm{~Hz}, 1 \mathrm{H}), 7.41(\mathrm{~s}, 1 \mathrm{H}), 7.28-7.29(\mathrm{~m}, 3 \mathrm{H}), 7.09-7.14(\mathrm{~m}$, $3 \mathrm{H}), 5.35(\mathrm{t}, J=6.6 \mathrm{~Hz}, 1 \mathrm{H}), 3.80(\mathrm{~s}, 3 \mathrm{H}), 3.48(\mathrm{~s}, 2 \mathrm{H}), 2.30(\mathrm{~s}, 3 \mathrm{H}), 2.28(\mathrm{~s}, 3 \mathrm{H}), 1.64(\mathrm{~d}, J=3.6 \mathrm{~Hz}, 3 \mathrm{H})$. ${ }^{13} \mathrm{C}-\mathrm{NMR}\left(125 \mathrm{MHz}, \mathrm{CDCl}_{3}\right) \delta$ 21.0, 21.6, 26.1, 33.9, 50.4, 53.5, 97.0, 124.4, 124.5, $125.3(2 \mathrm{C}), 129.2,129.4$ (2C), 135.6, 136.5, 137.6, 142.3, 142.7, 159.2, 168.9. IR (KBr): 3307, 3032, 2924, 2316, 1651, 1595, 1556, $1448,1263,1201 \mathrm{~cm}^{-1}$. MS (ESI): $m / z 322(\mathrm{M}+\mathrm{H})^{+}$.

Methyl 3-((1-(4-methoxyphenyl)ethyl)amino)-5-methyl-1H-indene-2-carboxylate (2e). Pale yellow solid (32\%, $156.3 \mathrm{mg}$ ). ${ }^{1} \mathrm{H}-\mathrm{NMR}\left(300 \mathrm{MHz}, \mathrm{CDCl}_{3}\right) \delta 8.23(\mathrm{~d}, J=7.2 \mathrm{~Hz}, 1 \mathrm{H}), 7.40(\mathrm{~s}, 1 \mathrm{H}), 7.25-7.34(\mathrm{~m}, 3 \mathrm{H}), 7.11(\mathrm{~d}$, $J=7.2 \mathrm{~Hz}, 1 \mathrm{H}), 6.86(\mathrm{~d}, J=8.4 \mathrm{~Hz}, 2 \mathrm{H}), 5.31-5.36(\mathrm{~m}, 1 \mathrm{H}), 3.79(\mathrm{~s}, 3 \mathrm{H}), 3.76(\mathrm{~s}, 3 \mathrm{H}), 3.48(\mathrm{~s}, 2 \mathrm{H}), 2.28(\mathrm{~s}$, $3 \mathrm{H}), 1.63(\mathrm{~d}, J=6.3 \mathrm{~Hz}, 3 \mathrm{H}) .{ }^{13} \mathrm{C}-\mathrm{NMR}\left(125 \mathrm{MHz}, \mathrm{CDCl}_{3}\right)$. IR (KBr): 3276, 2997, 2939, 2831, 1647, 1610, $1587,1506,1452,1329,1259,1190,1174,1092 \mathrm{~cm}^{-1}$. MS (ESI): $m / z 338(\mathrm{M}+\mathrm{H})^{+}$.

Methyl 3-((1-(naphthalen-1-yl)ethyl)amino)-1H-indene-2-carboxylate (2f). Yellow solid (42\%, $214.1 \mathrm{mg})$. ${ }^{1} \mathrm{H}-\mathrm{NMR}\left(300 \mathrm{MHz}, \mathrm{CDCl}_{3}\right) \delta 8.47(\mathrm{~d}, J=6.6 \mathrm{~Hz}, 1 \mathrm{H}), 8.14(\mathrm{~d}, J=8.4 \mathrm{~Hz}, 1 \mathrm{H}), 7.92(\mathrm{~d}, J=8.1 \mathrm{~Hz}$, $1 \mathrm{H}), 7.74(\mathrm{~d}, J=8.4 \mathrm{~Hz}, 1 \mathrm{H}), 7.52-7.67(\mathrm{~m}, 3 \mathrm{H}), 7.37-7.42(\mathrm{~m}, 2 \mathrm{H}), 7.29(\mathrm{~d}, J=7.8 \mathrm{~Hz}, 1 \mathrm{H}), 7.20(\mathrm{t}, J=$ $7.5 \mathrm{~Hz}, 1 \mathrm{H}), 6.91(\mathrm{t}, J=7.7 \mathrm{~Hz}, 1 \mathrm{H}), 6.07-6.17(\mathrm{~m}, 1 \mathrm{H}), 3.84(\mathrm{~s}, 3 \mathrm{H}), 3.56(\mathrm{~s}, 2 \mathrm{H}), 1.79(\mathrm{~d}, J=6.6 \mathrm{~Hz}, 3 \mathrm{H})$. ${ }^{13} \mathrm{C}-\mathrm{NMR}\left(125 \mathrm{MHz}, \mathrm{CDCl}_{3}\right) \delta 24.9$ 34.3, 50.5, 96.7, 122.0, 122.5, 123.3, 124.9, 125.6, 125.9, 126.3, 126.5, 127.7, 128.2, 129.3, 129.8, 134.0, 137.3, 140.6, 145.5, 159.0, 169.0. IR (KBr): 3292, 3062, 2966, 2951, 1747, $1655,1606,1568,1444,1529,1190 \mathrm{~cm}^{-1}$. MS (ESI): $m / z 344(\mathrm{M}+\mathrm{H})^{+}$. 
Methyl 5-methoxy-3-((1-(naphthalen-1-yl)ethyl)amino)-1H-indene-2-carboxylate (2g). Yellow solid (46\%, $250.4 \mathrm{mg}) .{ }^{1} \mathrm{H}-\mathrm{NMR}\left(300 \mathrm{MHz}, \mathrm{CDCl}_{3}\right) \delta 8.48(\mathrm{~d}, J=4.5 \mathrm{~Hz}, 1 \mathrm{H}), 8.16(\mathrm{~d}, J=8.1 \mathrm{~Hz}, 1 \mathrm{H}), 7.91(\mathrm{~d}, J=$ $7.8 \mathrm{~Hz}, 1 \mathrm{H}), 7.70-7.76(\mathrm{~m}, 2 \mathrm{H}), 7.50-7.63(\mathrm{~m}, 2 \mathrm{H}), 7.42(\mathrm{t}, J=7.5 \mathrm{~Hz}, 1 \mathrm{H}), 7.21(\mathrm{~d}, J=8.7 \mathrm{~Hz}, 1 \mathrm{H}), 6.73$ $(\mathrm{dd}, J=8.4,2.4 \mathrm{~Hz}, 1 \mathrm{H}), 6.58(\mathrm{~d}, J=2.4 \mathrm{~Hz}, 1 \mathrm{H}), 6.01-6.09(\mathrm{~m}, 1 \mathrm{H}), 3.85(\mathrm{~s}, 3 \mathrm{H}), 3.53(\mathrm{~d}, J=22.2 \mathrm{~Hz}$, $1 \mathrm{H}), 3.44(\mathrm{~d}, J=22.2 \mathrm{~Hz}, 1 \mathrm{H}), 2.71(\mathrm{~s}, 3 \mathrm{H}), 1.82(\mathrm{~d}, J=6.9 \mathrm{~Hz}, 3 \mathrm{H}) .{ }^{13} \mathrm{C}-\mathrm{NMR}\left(125 \mathrm{MHz}, \mathrm{CDCl}_{3}\right) \delta 33.6$, $50.5,50.6,54.3,97.7,106.6,116.9,121.9,122.7,125.2,125.7,126.2,126.5,127.6,129.3,129.6,134.0,137.6$, 138.1, 141.0, 158.1, 159.1, 168.9. IR (KBr): 3300, 3057, 2945, 2829, 1741, 1655, 1614, 1576, 1452, 1225, 1132, $1086 \mathrm{~cm}^{-1}$. MS (ESI): $m / z 374(\mathrm{M}+\mathrm{H})^{+}$.

Methyl 5,6-dimethoxy-3-((1-(naphthalen-1-yl)ethyl)amino)-1H-indene-2-carboxylate (2h). Yellow solid (45\%, $265.6 \mathrm{mg}) .{ }^{1} \mathrm{H}-\mathrm{NMR}\left(300 \mathrm{MHz}, \mathrm{CDCl}_{3}\right) \delta 8.54$ (br s, $\left.1 \mathrm{H}\right), \delta 8.17(\mathrm{~d}, J=8.7 \mathrm{~Hz}, 1 \mathrm{H}), 7.91(\mathrm{~d}, J=8.1 \mathrm{~Hz}$, $1 \mathrm{H}), 7.69-7.75(\mathrm{~m}, 2 \mathrm{H}), 7.50-7.63(\mathrm{~m}, 2 \mathrm{H}), 7.38-7.43(\mathrm{~m}, 1 \mathrm{H}), 6.85(\mathrm{~s}, 1 \mathrm{H}), 6.47(\mathrm{~s}, 1 \mathrm{H}), 5.96-6.05(\mathrm{~m}$, $1 \mathrm{H}), 3.84(\mathrm{~s}, 3 \mathrm{H}), 3.80(\mathrm{~s}, 3 \mathrm{H}), 3.53(\mathrm{~d}, J=22.2 \mathrm{~Hz}, 1 \mathrm{H}), 3.44(\mathrm{~d}, J=22.2 \mathrm{~Hz}, 1 \mathrm{H}) 2.61(\mathrm{~s}, 3 \mathrm{H}), 1.83(\mathrm{~d}, J$ $=6.3 \mathrm{~Hz}, 3 \mathrm{H}) .{ }^{13} \mathrm{C}-\mathrm{NMR}\left(125 \mathrm{MHz}, \mathrm{CDCl}_{3}\right) \delta 24.7,34.1,50.3,50.5,54.6,55.8,95.3,106.0,107.2,121.8$, 122.7, $125.7126 .2,126.5,127.6,129.1,129.4,129.5,133.9,139.1,140.9,147.4,149.8,159.7,168.6 . \mathrm{IR}(\mathrm{KBr})$ : $3300,2947,1739,1643,1595,1556,1448,1309,1252,1209 \mathrm{~cm}^{-1}$. MS (ESI): $m / z 404(\mathrm{M}+\mathrm{H})^{+}$.

Methyl 6-bromo-3-((1-(naphthalen-1-yl)ethyl)amino)-1H-indene-2-carboxylate (2i). Yellow solid (42\%, $262.9 \mathrm{mg}) .{ }^{1} \mathrm{H}-\mathrm{NMR}\left(300 \mathrm{MHz}^{\mathrm{CDCl}}{ }_{3}\right) \delta 8.41(\mathrm{br} \mathrm{d}, 1 \mathrm{H}), 8.10(\mathrm{~d}, J=8.7 \mathrm{~Hz}, 1 \mathrm{H}), 7.92(\mathrm{~d}, J=8.1 \mathrm{~Hz}$, $1 \mathrm{H}), 7.74(\mathrm{~d}, J=7.5 \mathrm{~Hz}, 1 \mathrm{H}), 7.50-7.65(\mathrm{~m}, 4 \mathrm{H}), 7.39(\mathrm{t}, J=7.8 \mathrm{~Hz}, 1 \mathrm{H}), 7.09(\mathrm{~d}, J=8.4 \mathrm{~Hz}, 1 \mathrm{H}), 7.01(\mathrm{~d}, J$ $=8.4 \mathrm{~Hz}, 1 \mathrm{H}), 6.00-6.05(\mathrm{~m}, 1 \mathrm{H}), 3.84(\mathrm{~s}, 3 \mathrm{H}), 3.53(\mathrm{~s}, 2 \mathrm{H}), 1.78(\mathrm{~d}, J=6.6 \mathrm{~Hz}, 3 \mathrm{H}) .{ }^{13} \mathrm{C}-\mathrm{NMR}(125 \mathrm{MHz}$, $\left.\mathrm{CDCl}_{3}\right) \delta 24.8,34.2,50.4,50.6,96.9,121.7,122.5,122.8,124.3,125.7,125.9,126.7,127.8,128.0,123.0,129.4$, 129.6, 134.0, 136.3, 140.3, 147.3, 158.0, 168.7. IR (KBr): 3296, 3059, 2939, 2858, 1739, 1658, 1610, 1560, $1452,1325,1255,1186 \mathrm{~cm}^{-1}$. MS (ESI): $m / z 422(\mathrm{M}+\mathrm{H})^{+}$.

Methyl 6-fluoro-3-((1-(naphthalen-1-yl)ethyl)amino)-1H-indene-2-carboxylate (2j). Yellow solid (43\%, $230.9 \mathrm{mg}) .{ }^{1} \mathrm{H}-\mathrm{NMR}\left(300 \mathrm{MHz}, \mathrm{CDCl}_{3}\right) \delta 8.47(\mathrm{~d}, J=6.9 \mathrm{~Hz}, 1 \mathrm{H}), 8.12(\mathrm{~d}, J=8.4 \mathrm{~Hz}, 1 \mathrm{H}), 7.92$ $(\mathrm{d}, J=8.1 \mathrm{~Hz}, 1 \mathrm{H}), 7.74(\mathrm{~d}, J=8.1 \mathrm{~Hz}, 1 \mathrm{H}), 7.60-7.66(\mathrm{~m}, 2 \mathrm{H}), 7.53-7.58(\mathrm{~m}, 1 \mathrm{H}), 7.37-7.42(\mathrm{~m}, 1 \mathrm{H}), 7.19$ $(\mathrm{dd}, J=8.7,5.1 \mathrm{~Hz}, 1 \mathrm{H}), 7.06(\mathrm{dd}, J=8.4,2.4 \mathrm{~Hz}, 1 \mathrm{H}), 6.59(\mathrm{td}, J=9.0,2.4 \mathrm{~Hz}, 1 \mathrm{H}), 6.00-6.09(\mathrm{~m}, 1 \mathrm{H})$, $3.84(\mathrm{~s}, 3 \mathrm{H}), 3.59(\mathrm{~d}, J=22.8 \mathrm{~Hz}, 1 \mathrm{H}), 3.50(\mathrm{~d}, J=22.8 \mathrm{~Hz}, 1 \mathrm{H}) 1.78(\mathrm{~d}, J=6.6 \mathrm{~Hz}, 3 \mathrm{H}) .{ }^{19} \mathrm{~F}-\mathrm{NMR}(282$ $\left.\mathrm{MHz}, \mathrm{CDCl}_{3}\right) \delta-113.3$ (s, 1F). ${ }^{13} \mathrm{C}-\mathrm{NMR}\left(125 \mathrm{MHz}, \mathrm{CDCl}_{3}\right) \delta 24.9,34.4,50.4,50.5,96.5,112.1(\mathrm{~d}, J=$ $22.5 \mathrm{~Hz}, 1 \mathrm{C}), 113.6(\mathrm{~d}, J=22.5 \mathrm{~Hz}, 1 \mathrm{C}), 121.8,122.5,124.4(\mathrm{~d}, J=8.8 \mathrm{~Hz}, 1 \mathrm{C}), 125.7,125.9,126.7,127.8$, 129.4, 129.7, 133.4, 134.0, 140.4, $148.2(\mathrm{~d}, J=8.8 \mathrm{~Hz}, 1 \mathrm{C}), 158.2,163.1(\mathrm{~d}, J=247.5 \mathrm{~Hz}, 1 \mathrm{C}), 168.8 . \mathrm{IR}(\mathrm{KBr})$ : $3296,3057,2945,1741,1655,1614,1576,1452,1225,1132,1086 \mathrm{~cm}^{-1}$. MS (ESI): $m / z 362(\mathrm{M}+\mathrm{H})^{+}$.

Ethyl 3-((1-(Naphthalen-1-Yl)ethyl)amino)-1H-Indene-2-Carboxylate (2k). Amine (2.0 mmol, 1.5 equiv) was added to a solution of $\beta$-ketoester $(1.35 \mathrm{mmol})$ and zinc acetate $(0.27 \mathrm{mmol}, 20 \mathrm{~mol} \%)$ in methanol under a nitrogen atmosphere, the reaction mixture was refluxed for $60 \mathrm{~h}$. After reaction, the mixture was concentrated under reduced pressure, and the residue was purified by column chromatography (ethyl acetate: hexane). Yellow solid (43\%, $205.6 \mathrm{mg}) .{ }^{1} \mathrm{H}-\mathrm{NMR}\left(300 \mathrm{MHz}, \mathrm{CDCl}_{3}\right) \delta 8.45(\mathrm{~d}, J=6.0 \mathrm{~Hz}$, $1 \mathrm{H}), 8.14(\mathrm{~d}, J=8.4 \mathrm{~Hz}, 1 \mathrm{H}), 7.91(\mathrm{~d}, J=7.8 \mathrm{~Hz}, 1 \mathrm{H}), 7.74(\mathrm{~d}, J=7.2 \mathrm{~Hz}, 1 \mathrm{H}), 7.52-7.68(\mathrm{~m}, 3 \mathrm{H}), 7.37-7.42$ $(\mathrm{m}, 2 \mathrm{H}), 7.29(\mathrm{~d}, J=8.1 \mathrm{~Hz}, 1 \mathrm{H}), 7.19(\mathrm{t}, J=7.5 \mathrm{~Hz}, 1 \mathrm{H}), 6.90(\mathrm{t}, J=7.8 \mathrm{~Hz}, 1 \mathrm{H}), 6.08-6.16(\mathrm{~m}, 1 \mathrm{H}), 4.31$ $(\mathrm{q}, J=7.1 \mathrm{~Hz}, 2 \mathrm{H}), 3.61(\mathrm{~d}, J=22.8 \mathrm{~Hz}, 1 \mathrm{H}), 3.53(\mathrm{~d}, J=23.1 \mathrm{~Hz}, 1 \mathrm{H}), 1.78(\mathrm{~d}, J=6.9 \mathrm{~Hz}, 3 \mathrm{H}), 1.38(\mathrm{t}, J=$ 7.2 Hz, 3H). ${ }^{13} \mathrm{C}-\mathrm{NMR}\left(125 \mathrm{MHz}, \mathrm{CDCl}_{3}\right) \delta 14.8,24.9,34.4,50.3,58.9,97.1,122.0,122.5,123.8,124.8$, $125.5,125.9,126.2,126.5,127.6,128.1,129.2,129.8,133.9,137.4,140.6,145.6,158.7,168.6 . \mathrm{IR}(\mathrm{KBr}): 3296$, $3059,2974,1739,1647,1610,1591,1564,1441,1259,1186,1120 \mathrm{~cm}^{-1}$. MS (ESI): $\mathrm{m} / z 358(\mathrm{M}+\mathrm{H})^{+}$.

\subsection{Representative Procedure for the Diastereoselective Difluoromethylthiolation}

\section{General Procedure}

Reagent $1 \mathbf{b}$ [24] ( $0.40 \mathrm{mmol}, 2.0$ equiv) was added to a solution of enamine ( $0.20 \mathrm{mmol}, 1.0$ equiv) and $\mathrm{CuBr}(0.04 \mathrm{mmol}, 20 \mathrm{~mol} \%)$ in toluene $(2.5 \mathrm{~mL})$ under a nitrogen atmosphere, and the reaction mixture was stirred at room temperature for $5 \mathrm{~h}$. $\mathrm{HCl}(1 \mathrm{M})$ was added to the reaction mixture which 
was then stirred for $12 \mathrm{~h}$. After that, the mixture was extracted with ethyl acetate two times, then washed with brine and dried by $\mathrm{Na}_{2} \mathrm{SO}_{4}$. The ethyl acetate was removed under reduced pressure and the residue was purified by column chromatography (ethyl acetate: hexane) or $\left(\mathrm{CH}_{2} \mathrm{Cl}_{2}\right.$ : hexane).

Methyl 2-((difluoromethyl)thio)-6-methyl-1-oxo-2,3-dihydro-1H-indene-2-carboxylate (3a) [23-25]. Yellow oil ( $24.2 \mathrm{mg}$, 45\%, 88\% ee). The ee value was determined by HPLC analysis using a Chiralpack IB3 column (hexane $/ \mathrm{PrOH}=98: 2$, flow rate: $0.5 \mathrm{~mL} / \mathrm{min}, \mathrm{t}_{\mathrm{R}}($ minor $)=18.8 \mathrm{~min}$ (integral $\left.=5.9 \%\right), \mathrm{t}_{\mathrm{R}}($ major) $=$ $21.8 \mathrm{~min}$ (integral = 94.1\%). ${ }^{1} \mathrm{H}-\mathrm{NMR}\left(300 \mathrm{MHz}, \mathrm{CDCl}_{3}\right) \delta 7.64(\mathrm{~s}, 1 \mathrm{H}), 7.52(\mathrm{~S}, 1 \mathrm{H}), 7.50(\mathrm{t}, J=55.7 \mathrm{~Hz}$ $1 \mathrm{H}), 7.36(\mathrm{~d}, J=7.6 \mathrm{~Hz}, 1 \mathrm{H}), 3.98(\mathrm{~d}, J=17.6 \mathrm{~Hz}, 1 \mathrm{H}), 3.81(\mathrm{~s}, 3 \mathrm{H}), 3.22(\mathrm{~d}, J=17.9 \mathrm{~Hz}, 1 \mathrm{H}), 2.43(\mathrm{~s}$, 3H). ${ }^{19} \mathrm{~F}-\mathrm{NMR}\left(282 \mathrm{MHz}, \mathrm{CDCl}_{3}\right) \delta-92.1(\mathrm{dd}, J=251.7,55.2 \mathrm{~Hz}, 1 \mathrm{~F}),-93.5(\mathrm{dd}, J=250.9,56.0 \mathrm{~Hz}, 1 \mathrm{~F})$. HRMS (ESI) $m / z$ Calcd: 309.0373 for $\mathrm{C}_{13} \mathrm{H}_{12} \mathrm{O}_{3} \mathrm{~F}_{2} \mathrm{SNa}(\mathrm{M}+\mathrm{Na})^{+}$Found: 309.0370.

Methyl 2-((difluoromethyl)thio)-1-oxo-2,3-dihydro-1H-indene-2-carboxylate (3b). Pale yellow solid (30.7 mg, $56 \%, 85 \%$ ee $) .[\alpha]^{25} \mathrm{D}=+6.6\left(c=0.77, \mathrm{CHCl}_{3}\right)$. The ee value was determined by HPLC analysis using a Chiralpack IB3 column (hexane $/ \mathrm{PrOH}=98: 2$, flow rate: $0.5 \mathrm{~mL} / \mathrm{min}, \mathrm{t}_{\mathrm{R}}($ minor) $=30.2 \mathrm{~min}$ (integral = $7.4 \%), \mathrm{t}_{\mathrm{R}}($ major $)=33.5 \mathrm{~min}$ (integral = 92.5\%). ${ }^{1} \mathrm{H}-\mathrm{NMR}\left(700 \mathrm{MHz}, \mathrm{CDCl}_{3}\right) \delta 7.84(\mathrm{~d}, J=7.6 \mathrm{~Hz}, 1 \mathrm{H})$, $7.70(\mathrm{~d}, J=7.6 \mathrm{~Hz}, 1 \mathrm{H}), 7.49(\mathrm{t}, J=52.2 \mathrm{~Hz}, 1 \mathrm{H}), 7.47(\mathrm{~s}, 3 \mathrm{H}), 7.44-7.50(\mathrm{~m}, 2 \mathrm{H}), 4.04(\mathrm{~d}, J=17.9 \mathrm{~Hz}$, $1 \mathrm{H}), 3.82(\mathrm{~s}, 3 \mathrm{H}), 3.27(\mathrm{~d}, J=17.9 \mathrm{~Hz}, 1 \mathrm{H}) .{ }^{19} \mathrm{~F}-\mathrm{NMR}\left(282 \mathrm{MHz}, \mathrm{CDCl}_{3}\right) \delta-92.0(\mathrm{dd}, J=250.9,56.0 \mathrm{~Hz}$, $1 \mathrm{~F}),-93.4(\mathrm{dd}, J=250.0,55.2 \mathrm{~Hz}, 1 \mathrm{~F}) .{ }^{13} \mathrm{C}-\mathrm{NMR}\left(125 \mathrm{MHz}, \mathrm{CDCl}_{3}\right) \delta 196.9,169.0,150.7,136.5,133.1$, $120.4(\mathrm{t}, J=271.1 \mathrm{~Hz}), 122.6,120.4,118.3,58.6,54.2,39.7 . \mathrm{IR}(\mathrm{KBr}): 3032,2966,1759,1720,1603,1464$, 1433, 1248, 1190, 1068, $1030 \mathrm{~cm}^{-1}$. HRMS (ESI) $m / z$ Calcd: 295.0216 for $\mathrm{C}_{12} \mathrm{H}_{10} \mathrm{O}_{3} \mathrm{~F}_{2} \mathrm{SNa}(\mathrm{M}+\mathrm{Na})^{+}$, Found 295.0228 .

Methyl 2-((difluoromethyl)thio)-6-methoxy-1-oxo-2,3-dihydro-1H-indene-2-carboxylate (3c) [24,26]. Yellow oil $\left(31.1 \mathrm{mg}, 51 \%, 90 \%\right.$ ee). $[\alpha]^{25} \mathrm{D}=-15.7\left(c=0.64, \mathrm{CHCl}_{3}\right)$. The ee value was determined by HPLC analysis using a Chiralpack IB3 column (hexane $/ \mathrm{PrOH}=95: 5$, flow rate: $0.5 \mathrm{~mL} / \mathrm{min}, \mathrm{t}_{\mathrm{R}}($ minor) $=$ $23.9 \mathrm{~min}$ (integral $=5.1 \%), \mathrm{t}_{\mathrm{R}}$ (major) $=25.9 \mathrm{~min}($ integral $=94.9 \%) .{ }^{1} \mathrm{H}-\mathrm{NMR}\left(300 \mathrm{MHz}, \mathrm{CDCl}_{3}\right) \delta 7.52$ $(\mathrm{t}, J=55.7 \mathrm{~Hz}, 1 \mathrm{H}), 7.25-7.38(\mathrm{~m}, 3 \mathrm{H}), 3.95(\mathrm{~d}, J=17.6 \mathrm{~Hz}, 1 \mathrm{H}), 3.86(\mathrm{~s}, 3 \mathrm{H}), 3.82(\mathrm{~s}, 3 \mathrm{H}), 3.20(\mathrm{~d}, J=$ 17.6 Hz, 1H). ${ }^{19} \mathrm{~F}-\mathrm{NMR}\left(282 \mathrm{MHz}, \mathrm{CDCl}_{3}\right) \delta-92.1(\mathrm{dd}, J=251.7,55.2 \mathrm{~Hz}, 1 \mathrm{~F}),-93.5(\mathrm{dd}, J=250.9$, $56.0 \mathrm{~Hz}, 1 \mathrm{~F})$. HRMS (ESI) $m / z$ Calcd: 325.0322 for $\mathrm{C}_{13} \mathrm{H}_{12} \mathrm{O}_{4} \mathrm{~F}_{2} \mathrm{NaS}(\mathrm{M}+\mathrm{Na})^{+}$Found: 325.0325 .

Methyl 2-((difluoromethyl)thio)-5,6-dimethoxy-1-oxo-2,3-dihydro-1H-indene-2-carboxylate (3d) [26]. Brown solid $\left(36.2 \mathrm{mg}, 54 \%, 94 \%\right.$ ee). $[\alpha]^{25} \mathrm{D}=-12.3\left(c=0.70, \mathrm{CHCl}_{3}\right)$. The ee value was determined by HPLC analysis using a Chiralpack OD-3 column (hexane $/ \mathrm{PrOH}=95: 5$, flow rate: $1.0 \mathrm{~mL} / \mathrm{min}, \mathrm{t}_{\mathrm{R}}($ minor) $=$ $27.2 \mathrm{~min}$ (integral $=3.2 \%), \mathrm{t}_{\mathrm{R}}$ (major) $=29.3 \mathrm{~min}$ (integral $\left.=96.8 \%\right) .{ }^{1} \mathrm{H}-\mathrm{NMR}\left(300 \mathrm{MHz}, \mathrm{CDCl}_{3}\right) \delta 7.52$ $(\mathrm{t}, J=55.7 \mathrm{~Hz}, 1 \mathrm{H}), 7.27(\mathrm{~s}, 1 \mathrm{H}), 6.88(\mathrm{~s}, 1 \mathrm{H}), 4.00(\mathrm{~s}, 3 \mathrm{H}), 3.93(\mathrm{~m}, 4 \mathrm{H}), 3.82(\mathrm{~s}, 3 \mathrm{H}), 3.21(\mathrm{~d}, J=17.6 \mathrm{~Hz}$, 1H). ${ }^{19} \mathrm{~F}-\mathrm{NMR}\left(282 \mathrm{MHz}, \mathrm{CDCl}_{3}\right) \delta-91.9(\mathrm{dd}, J=250.9,56.0 \mathrm{~Hz}, 1 \mathrm{~F}),-93.5(\mathrm{dd}, J=250.9,56.0 \mathrm{~Hz}, 1 \mathrm{~F})$. HRMS (ESI) $m / z$ Calcd: 355.0428 for $\mathrm{C}_{14} \mathrm{H}_{14} \mathrm{O}_{5} \mathrm{~F}_{2} \mathrm{SNa}(\mathrm{M}+\mathrm{Na})^{+}$Found: 355.0427 .

Methyl 5-bromo-2-((difluoromethyl)thio)-1-oxo-2,3-dihydro-1H-indene-2-carboxylate (3e). Brown solid (22.6 mg, 32\%, 88\% ee). M.p. 58.2-63.8 ${ }^{\circ} \mathrm{C} .[\alpha]^{25} \mathrm{D}=-2.5\left(c=1.1, \mathrm{CHCl}_{3}\right)$. The ee value was determined by HPLC analysis using a Chiralpack OD-3 column (hexane $/ \mathrm{PrOH}=95: 5$, flow rate: $1.0 \mathrm{~mL} / \mathrm{min}$, $\mathrm{t}_{\mathrm{R}}($ minor $)=10.6 \mathrm{~min}($ integral $=5.9 \%), \mathrm{t}_{\mathrm{R}}($ major $)=12.0 \mathrm{~min}($ integral $=94.0 \%) .{ }^{1} \mathrm{H}-\mathrm{NMR}(300 \mathrm{MHz}$, $\left.\mathrm{CDCl}_{3}\right) \delta 7.72-7.63(\mathrm{~m}, 3 \mathrm{H}), 7.45(\mathrm{t}, J=53.3 \mathrm{~Hz}, 1 \mathrm{H}), 4.03(\mathrm{~d}, J=18.5 \mathrm{~Hz}, 1 \mathrm{H}), 3.83(\mathrm{~s}, 3 \mathrm{H}), 3.27(\mathrm{~d}, J$ $=17.9 \mathrm{~Hz}, 1 \mathrm{H}) .{ }^{19} \mathrm{~F}-\mathrm{NMR}\left(282 \mathrm{MHz}, \mathrm{CDCl}_{3}\right) \delta-91.9(\mathrm{dd}, J=250.9,56.0 \mathrm{~Hz}, 1 \mathrm{~F}),-93.5(\mathrm{dd}, J=250.0$, $55.2 \mathrm{~Hz}, 1 \mathrm{~F}) .{ }^{13} \mathrm{C}-\mathrm{NMR}\left(125 \mathrm{MHz}, \mathrm{CDCl}_{3}\right) \delta 195.7,168.5,152.2,132.5,132.2,132.0,129.8,127.1,120.3(\mathrm{t}, J$ $=271.6 \mathrm{~Hz}), 58.768,54.4,39.3$. IR (KBr): 2958, 1747, 1709, 1591, 1425, 1317, 1259, 1209, 1057, $1030 \mathrm{~cm}^{-1}$. HRMS (ESI) $m / z$ Calcd: 372.9322 for $\mathrm{C}_{12} \mathrm{H}_{9} \mathrm{O}_{3} \mathrm{~F}_{2} \mathrm{NaSBr}(\mathrm{M}+\mathrm{Na})^{+}$Found: 372.9309 .

Methyl 2-((difluoromethyl)thio)-5-fluoro-1-oxo-2,3-dihydro-1H-indene-2-carboxylate (3f). Yellow oil (36.5 mg, $63 \%, 86 \%$ ee $) .[\alpha]^{25} \mathrm{D}=+6.5\left(c=0.77, \mathrm{CHCl}_{3}\right)$. The ee value was determined by HPLC analysis using a Chiralpack OD-3 column (hexane $/ \mathrm{PrOH}=95: 5$, flow rate: $1.0 \mathrm{~mL} / \mathrm{min}, \mathrm{t}_{\mathrm{R}}$ (minor) $=11.8 \mathrm{~min}$ (integral $=6.8 \%), \mathrm{t}_{\mathrm{R}}$ (major) $=13.3 \mathrm{~min}$ (integral = 93.2\%). ${ }^{1} \mathrm{H}-\mathrm{NMR}\left(300 \mathrm{MHz}, \mathrm{CDCl}_{3}\right) \delta 7.86(\mathrm{dd}, J=8.2,5.3 \mathrm{~Hz}$, $1 \mathrm{H}), 7.48(\mathrm{t}, J=55.5 \mathrm{~Hz}, 1 \mathrm{H}), 7.15-7.21(\mathrm{~m}, 2 \mathrm{H}), 4.05(\mathrm{~d}, J=17.9 \mathrm{~Hz}, 1 \mathrm{H}), 3.82(\mathrm{~s}, 3 \mathrm{H}), 3.28(\mathrm{~d}, J=17.9 \mathrm{~Hz}$, 
1H). ${ }^{19} \mathrm{~F}-\mathrm{NMR}\left(282 \mathrm{MHz}, \mathrm{CDCl}_{3}\right) \delta-91.9(\mathrm{dd}, J=250.9,56.0 \mathrm{~Hz}, 1 \mathrm{~F}),-93.5(\mathrm{dd}, J=250.0,55.2 \mathrm{~Hz}, 1 \mathrm{~F})$, -99.6 (dd, $J=13.8 \mathrm{~Hz}, 1 \mathrm{~F}) .{ }^{13} \mathrm{C}-\mathrm{NMR}\left(125 \mathrm{MHz}, \mathrm{CDCl}_{3}\right) \delta 194.9,168.4,168.0(\mathrm{~d}, J=259.8 \mathrm{~Hz}), 153.6(\mathrm{~d}$, $J=10.9 \mathrm{~Hz}), 128.3(\mathrm{~d}, J=10.9 \mathrm{~Hz}), 117.2(\mathrm{~d}, J=23.6 \mathrm{~Hz}), 113.2(\mathrm{~d}, J=22.7 \mathrm{~Hz}), 58.8,54.2,39.4$. IR (neat): 3074, 2958, 1747, 1720, 1618, 1595, 1429, 1255, 1068, $1041 \mathrm{~cm}^{-1}$. HRMS (ESI) $\mathrm{m} / z$ Calcd: 313.0122 for $\mathrm{C}_{12} \mathrm{H}_{9} \mathrm{O}_{3} \mathrm{~F}_{3} \mathrm{NaS}(\mathrm{M}+\mathrm{Na})^{+}$Found: 313.0120.

Ethyl 2-((difluoromethyl)thio)-1-oxo-2,3-dihydro-1H-indene-2-carboxylate (3g). Yellow oil (35 mg, 61\%, 85\% ee). $[\alpha]^{25} \mathrm{D}=+3.8\left(c=0.46, \mathrm{CHCl}_{3}\right)$. The ee value was determined by HPLC analysis using a Chiralpack IB3 column (hexane $/ \mathrm{PrOH}=99: 1$, flow rate: $1.0 \mathrm{~mL} / \mathrm{min}, \mathrm{t}_{\mathrm{R}}($ minor) $=24.5 \mathrm{~min}$ (integral $=7.6 \%), \mathrm{t}_{\mathrm{R}}$ (major) $=27.3 \mathrm{~min}\left(\right.$ integral = 92.4\%). ${ }^{1} \mathrm{H}-\mathrm{NMR}\left(300 \mathrm{MHz}, \mathrm{CDCl}_{3}\right) \delta 7.85(\mathrm{~d}, J=8.2 \mathrm{~Hz}, 1 \mathrm{H}), 7.70(\mathrm{~d}, J=$ $15.9 \mathrm{~Hz}, 1 \mathrm{H}), 7.53(\mathrm{t}, J=51.2 \mathrm{~Hz}, 1 \mathrm{H}), 7.45-7.50(\mathrm{~m}, 2 \mathrm{H}), 4.29(\mathrm{q}, J=7.2 \mathrm{~Hz}, 2 \mathrm{H}), 4.04(\mathrm{~d}, J=17.9 \mathrm{~Hz}$, $1 \mathrm{H}), 3.25(\mathrm{~d}, J=17.9 \mathrm{~Hz}, 1 \mathrm{H}), 1.29(\mathrm{t}, J=7.2 \mathrm{~Hz}, 3 \mathrm{H}) .{ }^{19} \mathrm{~F}-\mathrm{NMR}\left(282 \mathrm{MHz}, \mathrm{CDCl}_{3}\right) \delta-91.9(\mathrm{dd}, J=250.9$, $56.0 \mathrm{~Hz}, 1 \mathrm{~F}),-93.3(\mathrm{dd}, J=250.0,56.9 \mathrm{~Hz}, 1 \mathrm{~F}) .{ }^{13} \mathrm{C}-\mathrm{NMR}\left(125 \mathrm{MHz}, \mathrm{CDCl}_{3}\right) \delta 197.1,168.4,150.7,136.5$, $133.2,128.8,126.4,126.0,120.5(t, J=270.7 \mathrm{~Hz}), 63.6,58.5,39.6,14.1$. IR (neat): 2985, 1739, 1720, 1606, $1468,1271,1244,1213,1182,1065,1034 \mathrm{~cm}^{-1}$. HRMS (ESI) $m / z$ Calcd: 309.0373 for $\mathrm{C}_{13} \mathrm{H}_{12} \mathrm{O}_{3} \mathrm{~F}_{2} \mathrm{NaS}_{(\mathrm{M}}$ $+\mathrm{Na})^{+}$Found: 309.0351 .

Methyl 2-((difluoromethyl)thio)-2-Methyl-3-Oxo-3-Phenylpropanoate (3h). Colorless oil (19.5 mg, 36\%, 12\% ee). The ee value was determined by HPLC analysis using a Chiralpack OD-3 column (hexane $/ \mathrm{PrOH}$ $=99: 1$, flow rate: $0.53 \mathrm{~mL} / \mathrm{min}, \mathrm{t}_{\mathrm{R}}$ (minor) $=40.2 \mathrm{~min}$ (integral $\left.=43.8 \%\right), \mathrm{t}_{\mathrm{R}}$ (major) $=45.3 \mathrm{~min}$ (integral $=52.5 \%) .{ }^{1} \mathrm{H}-\mathrm{NMR}\left(300 \mathrm{MHz}, \mathrm{CDCl}_{3}\right) \delta 7.91(\mathrm{~d}, J=7.4 \mathrm{~Hz}, 2 \mathrm{H}), 7.56-7.61(\mathrm{~m}, 1 \mathrm{H}), 7.43-7.48(\mathrm{~m}, 2 \mathrm{H})$, $6.89(\mathrm{t}, J=55.9 \mathrm{~Hz}, 1 \mathrm{H}), 3.73(\mathrm{~s}, 3 \mathrm{H}), 1.97(\mathrm{~s}, 3 \mathrm{H}) .{ }^{19} \mathrm{~F}-\mathrm{NMR}\left(282 \mathrm{MHz}, \mathrm{CDCl}_{3}\right) \delta-92.7(\mathrm{~d}, J=55.2 \mathrm{~Hz}$, 2F). HRMS (ESI) $m / z: 297(\mathrm{M}+\mathrm{Na})^{+}$.

\section{Conclusions}

In summary, we have described the first asymmetric electrophilic difluoromethylthiolation of $\beta$-keto esters by means of a difluoromethanesulfonyl hypervalent iodonium ylide. The traceless use of chiral amines as chiral auxiliary allowed the synthesis of enantioenriched indanone-based $\alpha-\mathrm{SCF} \mathrm{F}_{2} \mathrm{H}$ $\beta$-keto esters in up to $93 \%$ ee value. We believe that this synthetic approach to enantiomerically enriched indanone scaffolds will create interest for the design of new biologically attractive drug candidates having $\alpha-\mathrm{SCF}_{2} \mathrm{H}$ indanone moiety. While tetralone-type and acyclic substrates failed to react efficiently, the improvement of the results could be theoretically possible by using chiral amines with electron withdrawing groups. This investigation is ongoing in our laboratory.

Supplementary Materials: The following are available online, ${ }^{1} \mathrm{H},{ }^{13} \mathrm{C}$ and ${ }^{19} \mathrm{~F}$ NMR spectra of compounds 3 and HPLC data of compounds 3.

Author Contributions: N.S. conceived and designed the experiments and directed the project; S.G., O.M. and H.C. performed the experiments; S.G., O.M. and H.C. and Y.S. analyzed the data; D.C. contributed to critical discussion and presentation of the results; Y.S. checked the experimental details; D.C. and N.S. wrote the paper.

Funding: This work was partially supported by ACT-C from the JST (JPMJCR12Z7), the CNRS Project International de Coopération Scientifique (PICS) N$^{\circ} 7262$ and JSPS KAKENHI Grant-in-Aid for Scientific Research (B) Grant Number $18 \mathrm{H} 02553$.

Acknowledgments: We thank Juhua Group Corporation for the gift of sodium difluoromethanesulfinate.

Conflicts of Interest: The authors declare no conflicts of interest.

\section{References and Notes}

1. Toulgoat, F.; Alazet, S.; Billard, T. Direct trifluoromethylthiolation reactions: The "renaissance" of an old concept. Eur. J. Org. Chem. 2014, 2014, 2415-2428. [CrossRef]

2. $\mathrm{Xu}, \mathrm{X} .-\mathrm{H} . ;$ trifluoromethylation, trifluoromethylthiolation, triflylation, and related reactions. Chem. Rev. 2015, 115, 731-764. [CrossRef] 
3. Xiong, H.-Y.; Pannecoucke, X.; Besset, T. Recent advances in the synthesis of $\mathrm{SCF}_{2} \mathrm{H}$ - and $\mathrm{SCF}_{2} \mathrm{FG}_{\text {-containing }}$ molecules. Chem. Eur. J. 2016, 22, 16734-16749. [CrossRef]

4. Barata-Vallejo, S.; Bonesi, S.; Postigo, A. Late stage trifluoromethylthiolation strategies for organic compounds. Org. Biomol. Chem. 2016, 14, 7150-7182. [CrossRef]

5. Erickson, J.A.; McLoughlin, J.I. Hydrogen bond donor properties of the difluoromethyl group. J. Org. Chem. 1995, 60, 1626-1631. [CrossRef]

6. Chachignon, H.; Kondrashov, E.V.; Cahard, D. Asymmetric construction of chiral carbon centers featuring the $\mathrm{SCF}_{3}$ motif: Direct trifluoromethylthiolation versus building block approach. Fluorine Notes 2017, 113, 5. [CrossRef]

7. Shao, X.; Wang, X.; Yang, T.; Lu, L.; Shen, Q. An electrophilic hypervalent iodine reagent for trifluoromethylthiolation. Angew. Chem. Int. Ed. 2013, 52, 3457-3460. [CrossRef] [PubMed]

8. Wang, X.; Yang, T.; Cheng, X.; Shen, Q. Enantioselective electrophilic trifluoromethylthiolation of $\beta$-ketoesters: A case of reactivity and selectivity bias for organocatalysis. Angew. Chem. Int. Ed. 2013, 52, 12860-12864. [CrossRef]

9. Bootwicha, T.; Liu, X.; Pluta, R.; Atodiresei, I.; Rueping, M. N-trifluoromethylthiophthalimide: A stable electrophilic $\mathrm{SCF}_{3}$-reagent and its application in the catalytic asymmetric trifluoromethylsulfenylation. Angew. Chem. Int. Ed. 2013, 52, 12856-12859. [CrossRef]

10. Deng, Q.-H.; Rettenmeier, C.; Wadepohl, H.; Gade, L.H. Copper-boxmi complexes as highly enantioselective catalysts for electrophilic trifluoromethylthiolations. Chem. Eur. J. 2014, 20, 93-97. [CrossRef]

11. Zhu, X.L.; Xu, J.H.; Cheng, D.J.; Zhao, L.J.; Liu, X.Y.; Tan, B. In situ generation of electrophilic trifluoromethylthio reagents for enantioselective trifluoromethylthiolation of oxindoles. Org. Lett. 2014, 16, 2192-2195. [CrossRef] [PubMed]

12. Yang, T.; Shen, Q.; Lu, L. Cinchona alkaloid-catalyzed enantioselective trifluoromethylthiolation of oxindoles. Chin. J. Chem. 2014, 32, 678-680. [CrossRef]

13. Rueping, M.; Liu, X.; Bootwicha, T.; Pluta, R.; Merkens, C. Catalytic enantioselective trifluoromethylthiolation of oxindoles using shelf-stable $N$-(trifluoromethylthio)phthalimide and a cinchona alkaloid catalyst. Chem. Commun. 2014, 50, 2508-2511. [CrossRef] [PubMed]

14. Liu, X.; An, R.; Zhang, X.; Luo, J.; Zhao, X. Enantioselective trifluoromethylthiolating lactonization catalyzed by an indane-based chiral sulfide. Angew. Chem. Int. Ed. 2016, 55, 5846-5850. [CrossRef] [PubMed]

15. Hu, L.; Wu, M.; Wan, H.; Wang, J.; Wang, G.; Guoa, H.; Sun, S. Efficient catalytic $\alpha$-trifluoromethylthiolation of aldehydes. New. J. Chem. 2016, 40, 6550-6553. [CrossRef]

16. Li, M.; Xue, X.-S.; Cheng, J.-P. Mechanism and origins of stereoinduction in natural cinchona alkaloid catalyzed asymmetric electrophilic trifluoromethylthiolation of $\beta$-keto esters with $\mathrm{N}$-trifluoromethylthiophthalimide as electrophilic $\mathrm{SCF}_{3}$ source. ACS Catal. 2017, 7, 7977-7986. [CrossRef]

17. Zeng, J.L.; Chachignon, H.; Ma, J.A.; Cahard, D. Nucleophilic trifluoromethylthiolation of cyclic sulfamidates: Access to chiral $\beta$ - and $\gamma-\mathrm{SCF}_{3}$ amines and $\alpha$-amino esters. Org. Lett. 2017, 19, 1974-1977. [CrossRef] [PubMed]

18. Liu, X.; Liang, Y.; Ji, J.; Luo, J.; Zhao, X. Chiral selenide-catalyzed enantioselective allylic reaction and intermolecular difunctionalization of alkenes: Efficient construction of ${\mathrm{C}-\mathrm{SCF}_{3}}_{3}$ stereogenic molecules. J. Am. Chem. Soc. 2018, 140, 4782-4786. [CrossRef]

19. Jin, M.Y.; Li, J.; Huang, R.; Zhou, Y.; Chung, L.W.; Wang, J. Catalytic asymmetric trifluoromethylthiolation of carbonyl compounds via a diastereo and enantioselective $\mathrm{Cu}$-catalyzed tandem reaction. Chem. Commun. 2018, 54, 4581-4584. [CrossRef]

20. Gelat, F.; Poisson, T.; Biju, A.T.; Pannecoucke, X.; Besset, T. Trifluoromethylthiolation of $\alpha$-chloroaldehydes: Access to quaternary $\mathrm{SCF}_{3}$-containing centers. Eur. J. Org. Chem. 2018, 2018, 3693-3696. [CrossRef]

21. Chachignon, H.; Kondrashov, E.V.; Cahard, D. Diastereoselective electrophilic trifluoromethylthiolation of chiral oxazolidinones: Access to enantiopure $\alpha-\mathrm{SCF}_{3}$ alcohols. Adv. Synth. Catal. 2018, 360, 965-971. [CrossRef]

22. Luo, J.; Cao, Q.; Cao, X.; Zhao, X. Selenide-catalyzed enantioselective synthesis of trifluorome- thylthiolated tetrahydronaphthalenes by merging desymmetrization and trifluoromethylthiolation. Nat. Commun. 2018, 9, 527. [CrossRef] [PubMed] 
23. Kondo, H.; Maeno, M.; Sasaki, K.; Guo, M.; Hashimoto, M.; Shiro, M.; Shibata, N. Synthesis of chiral nonracemic $\alpha$-difluoromethylthio compounds with tetrasubstituted stereogenic centers via a palladium-catalyzed decarboxylative asymmetric allylic alkylation. Org. Lett. 2018, 20, 7044-7048. [CrossRef]

24. Arimori, S.; Matsubara, O.; Takada, M.; Shiro, M.; Shibata, N. Difluoromethanesulfonyl hypervalent iodonium ylides for electrophilic difluoromethylthiolation reactions under copper catalysis. R. Soc. Open Sci. 2016, 3, 160102. [CrossRef] [PubMed]

25. Huang, Z.; Matsubara, O.; Jia, S.; Tokunaga, E.; Shibata, N. Difluoromethylthiolation of phenols and related compounds with a $\mathrm{HF}_{2} \mathrm{CSO}_{2} \mathrm{Na} / \mathrm{Ph}_{2} \mathrm{PCl} / \mathrm{Me}_{3} \mathrm{SiCl}$ system. Org. Lett. 2017, 19, 934-937. [CrossRef] [PubMed]

26. Zhu, D.; Gu, Y.; Lu, L.; Shen, Q. N-difluoromethylthiophthalimide: A shelf-stable, electrophilic reagent for difluoromethylthiolation. J. Am. Chem. Soc. 2015, 137, 10547. [CrossRef] [PubMed]

27. Christoffers, J.; Baro, A.; Werner, T. $\alpha$-Hydroxylation of $\beta$-dicarbonyl compounds. Adv. Synth. Catal. 2004, 346, 143-151. [CrossRef]

28. Toullec, P.Y.; Bonaccorsi, C.; Mezzetti, A.; Togni, A. Expanding the scope of asymmetric electrophilic atom-transfer reactions: Titanium- and ruthenium-catalyzed hydroxylation of $\beta$-ketoesters. Proc. Natl. Acad. Sci. USA 2004, 101, 5810-5814. [CrossRef]

29. Christoffers, J.; Werner, T.; Frey, W.; Baro, A. Straightforward synthesis of (R)-(-)-kjellmanianone. Chem. Eur. J. 2004, 10, 1042-1045. [CrossRef]

30. Ishimaru, T.; Shibata, N.; Nagai, J.; Nakamura, S.; Toru, T.; Kanemasa, S. Lewis acid-catalyzed enantioselective hydroxylation reactions of oxindoles and $\beta$-keto esters using DBFOX ligand. J. Am. Chem. Soc. 2006, 128, 16488-16489. [CrossRef]

31. Lu, M.; Zhu, D.; Lu, Y.; Zeng, X.; Tan, B.; Xu, Z.; Zhong, G. Chiral brønsted acid-catalyzed enantioselective $\alpha$-hydroxylation of $\beta$-dicarbonyl compounds. J. Am. Chem. Soc. 2009, 131, 4562-4563. [CrossRef] [PubMed]

32. Li, J.; Chen, G.; Wang, Z.; Zhang, R.; Zhang, X.; Ding, K. Spiro-2,2'-bichroman-based bisoxazoline (SPANbox) ligands for $\mathrm{Zn}^{\mathrm{II}}$-catalyzed enantioselective hydroxylation of $\beta$-keto esters and 1,3-diester. Chem. Sci. 2011, 2 , 1141-1144. [CrossRef]

33. Lian, M.; Li, Z.; Cai, Y.; Meng, Q.; Gao, Z. Enantioselective photooxygenation of $\beta$-keto esters by chiral phase-transfer catalysis using molecular oxygen. Chem. Asian J. 2012, 7, 2019-2023. [CrossRef] [PubMed]

34. Zou, L.; Wang, B.; Mu, H.; Zhang, H.; Song, Y.; Qu, J. Development of tartaric acid derived chiral guanidines and their application to catalytic enantioselective $\alpha$-hydroxylation of $\beta$-dicarbonyl compounds. Org. Lett. 2013, 15, 3106-3109. [CrossRef] [PubMed]

35. Racemic products $\mathbf{3}$ were prepared according to ref $\mathbf{2 4}$ and used as reference for ee determination by HPLC analysis.

36. Recovery of chiral amine auxiliary: In the synthesis of $\mathbf{3 d}$ (3.2), water phase of the extraction was basified with $\mathrm{NaOH}$ aq to $\mathrm{pH} 14$, and it was extracted with $\mathrm{CH}_{2} \mathrm{Cl}_{2}$ three times. The combined organic phase was dried by $\mathrm{Na}_{2} \mathrm{SO}_{4}$ and concentrated in vacuo to give the chiral amine, (R)-1-(naphthalen-1-yl)ethan-1-amine in $25 \%$ yield.

37. Yang, Y.-D.; Azuma, A.; Tokunaga, E.; Yamasaki, M.; Shiro, M.; Shibata, N. Trifluoromethanesulfonyl hypervalent iodonium ylide for copper-catalyzed trifluoromethylthiolation of enamines, indoles, and $\beta$-keto esters. J. Am. Chem. Soc. 2013, 135, 8782-8785. [CrossRef] [PubMed]

38. Zhang, Z.-H.; Yin, L.; Wang, Y.-M. General and efficient method for the preparation of $\beta$-enamino ketones and esters catalyzed by indium tribromide. Adv. Synth. Catal. 2006, 348, 184-190. [CrossRef]

39. Zhao, M.; Wang, F.; Li, X. Cross-dehydrogenative coupling between enamino esters and ketones: Synthesis of tetrasubstituted pyrroles. Org. Lett. 2012, 14, 1412-1415. [CrossRef]

Sample Availability: Samples of the compounds 1a, $\mathbf{1 b}, \mathbf{3}$ are available from the authors. 